\title{
The MORPHEUS III protein crystallization screen: at the frontier of drug discovery
}

Gorrec F, MRC Laboratory of Molecular Biology (Cambridge, UK)

The original MORPHEUS screen has proven to be a very efficient protein crystallization screen in the long term for a broad variety of protein samples, and the first follow-up screen, MORPHEUS II starts to have an impact for many research groups. The main ideas behind the formulation of MORPHEUS III were the same as originally:

- Increase the chances of crystal nucleation and growth by integrating mixes of additives that can act as stabilizers, cross-linkers, etc.

- Follow systematic approaches to select the reagents and formulate the screen, such as the integration of ligands that are highly represented in the PDB and a $3 \mathrm{D}$ grid formulation.

- Consider pragmatic ways to facilitate structure solution. For example, the screening of cryoprotectants and flash-freezing crystals are simplified.

The novelty in MORPHEUS III is the selection of small drug-like compounds as additives (average MW $=248 \mathrm{Da}$ ). To some extend, the approach can be compared to fragment-based lead discovery. The primarily aim however is to obtain novel macromolecular crystals (with or without ligand observed in the structures).

The final formulation of the new 96-condition crystallization screen integrates 44 compounds overall, divided into 8 mixes of additives. Each mix of additives is combined with 4 cryoprotected precipitant mixes and 3 buffer systems to form the $3 \mathrm{D}$ grid. 